\title{
openheart Cardiac mechanics and dysfunction with anthracyclines in the community: results from the PREDICT study
}

\author{
Hari K Narayan, ${ }^{1}$ Wei Wei, ${ }^{2}$ Ziding Feng, ${ }^{2}$ Daniel Lenihan, ${ }^{3}$ Ted Plappert, ${ }^{4}$ \\ Virginia Englefield, ${ }^{4}$ Michael Fisch, ${ }^{5}$ Bonnie $\mathrm{Ky}^{4,6}$
}

To cite: Narayan HK, Wei W, Feng Z, et al. Cardiac mechanics and dysfunction with anthracyclines in the community: results from the PREDICT study. Open Heart 2017:4:e000524. doi:10.1136/openhrt-2016000524

- Additional material is available. To view please visit the journal online (http://dx. doi.org/10.1136/openhrt2016-000524).

Received 24 August 2016 Revised 2 September 2016 Accepted 20 September 2016

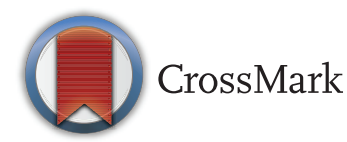

For numbered affiliations see end of article.

Correspondence to

Dr Bonnie Ky;

bonnie.ky@uphs.upenn.edu

\section{ABSTRACT}

Background: Our objective was to determine the relevance of changes in myocardial mechanics in diagnosing and predicting cancer therapeutics-related cardiac dysfunction (CTRCD) in a community-based population treated with anthracyclines.

Methods: Quantitative measures of cardiac mechanics were derived from 493 echocardiograms in 165 participants enrolled in the PREDICT study (A Multicenter Study in Patients Undergoing AnthRacycline-Based Chemotherapy to Assess the Effectiveness of Using Biomarkers to Detect and Identify Cardiotoxicity and Describe Treatment). Echocardiograms were obtained primarily at baseline (prior to anthracyclines), 6 and 12 months. Predictors included changes in strain; strain rate; indices of contractile function derived from the end-systolic pressure-volume relationship (end-systolic elastance (Ees $s_{\mathrm{sb}}$ ) and the left ventricular (LV) volume at an endsystolic pressure of $\left.100 \mathrm{~mm} \mathrm{Hg}\left(\mathrm{V}_{100}\right)\right)$; total arterial load (effective arterial elastance (Ea)) and ventriculararterial coupling $\left(\mathrm{Ea} / \mathrm{Ees}_{\mathrm{sb}}\right)$. Logistic regression models determined the diagnostic and prognostic associations of changes in these measures and CTRCD, defined as a LV ejection fraction decline $\geq 10$ to $<50 \%$.

Results: By 12 months, 31 participants developed CTRCD. Longitudinal and circumferential strain and strain rate, $\mathrm{V}_{100}$, Ea, and $\mathrm{Ea} / \mathrm{Ees}_{\mathrm{sb}}$ each demonstrated significant diagnostic associations, with a $1-7 \%$ increased odds of CTRCD $(p<0.05)$. Changes in longitudinal strain rate (area under the curve (AUC) $0.719(95 \% \mathrm{Cl} 0.595$ to 0.843$)$ ), $\mathrm{V}_{100}$ (AUC 0.796 $(95 \% \mathrm{Cl} 0.686$ to 0.903$))$ and Ea (AUC $0.742(95 \% \mathrm{Cl}$ 0.632 to 0.852$)$ ) from baseline to 6 months were individually predictive of CTRCD at 12 months.

Conclusions: Changes in non-invasively derived measures of myocardial mechanics are diagnostic and predictive of cardiac dysfunction with anthracycline chemotherapy in community populations. Our findings support the non-invasive assessment of measures of myocardial mechanics more broadly in clinical practice and emphasise the role of serial assessments of these measures during and after cardiotoxic cancer therapy. Trial registration number: NCT01032278;

Pre-results.

\section{INTRODUCTION}

Anthracyclines are widely used in the treatment of solid tumours and haematologic

\section{KEY QUESTIONS}

What is already known about this subject?

- Anthracycline chemotherapy carries a substantial risk of cardiac dysfunction and heart failure. A body of literature suggests that non-invasively derived measures of cardiac mechanics, such as strain, may predict subsequent cardiac dysfunction in tertiary care populations.

What does this study add?

- This study highlights the importance of understanding the changes in measures of mechanics with anthracycline chemotherapy; provides support for the use of ventricular-arterial coupling in cardio-oncology risk prediction and enhances the generalisability of the non-invasive assessment of mechanics to community-based oncology populations.

How might this impact on clinical practice?

- This study provides support for the use of noninvasive assessment of measures of myocardial mechanics more broadly in clinical practice and emphasises the role of serial assessments of these measures during and after cardiotoxic cancer therapy.

malignancies and have led to important survival gains. However, these agents can result in cardiovascular toxicity, including left ventricular (LV) dysfunction, heart failure (HF) and increased cardiovascular mortality. ${ }^{1}{ }_{2}$ Doxorubicin-induced declines in LV ejection fraction (EF) can occur in 15-17\% of patients, and $2-3 \%$ suffer from severe $\mathrm{HF}^{3}$ Early identification of patients at the highest cardiovascular risk could allow for targeted strategies to prevent these potential complications, and recent studies suggest that echocardiographic measures of mechanics may facilitate diagnosis and prognosis of subsequent cardiotoxicity. ${ }^{4-6}$ However, the relative utility of different measures and their usefulness in community-based oncology populations remain unclear.

$\mathrm{EF}$ is the most accepted measure in diagnosing cancer therapeutics-related cardiac 
dysfunction (CTRCD) and has been shown to be a valid prognostic marker in multiple cardiovascular disease populations. ${ }^{4}{ }^{7}$ However, EF may be relatively insensitive to early changes in cardiac function and is a summary measure of cardiac function, size and ventricular-arterial (VA) interaction. ${ }^{8} 9$ Abnormalities in strain, a measure of myocardial deformation, precede and are predictive of subsequent declines in EF and cardiovascular events across HF populations. ${ }^{5} 1011$ Other noninvasively derived measures of contractile function that may be useful in predicting CTRCD include those that describe the end-systolic pressure-volume relationship (ESPVR), which provide a load-independent measure of contractile function. The ESPVR can be defined by the slope (end-systolic elastance (Ees)), and the intercept $\left(\mathrm{V}_{0}\right)$, which is a measure of $\mathrm{LV}$ volume at a theoretical systolic pressure of $0 \mathrm{~mm} \mathrm{Hg}$. $\mathrm{V}_{0}$ is an extrapolated value obtained at a non-physiologic pressure, and thus the end-systolic volume at a pressure of $100 \mathrm{~mm} \mathrm{Hg}\left(\mathrm{V}_{100}\right)$ can also be described. Effective arterial elastance (Ea), the negative slope joining the end-systolic pressurevolume point to the point on the volume axis at enddiastole, measures arterial load. The net interaction of the ventricular and arterial system, indexed by the ratio $\mathrm{Ea} / \mathrm{Ees}$, is a measure of cardiovascular performance and efficiency. ${ }^{9}{ }^{12}$ Although there has been limited study of these indices in cardio-oncology, they are predictive of cardiac events in HF, and recent data from our own group suggest that these parameters have predictive utility in participants with breast cancer treated with doxorubicin and/or trastuzumab. ${ }^{9}{ }^{13}$ Despite the promise of these measures, their relevance in patients undergoing anthracycline chemotherapy remains incompletely understood. Additionally, the generalisability of their use to community-based oncology populations has not been defined.

Our overall objective was to determine the utility of these measures of myocardial mechanics to identify CTRCD in a community-based cardio-oncology cohort over a 12-month time period. We evaluated the diagnostic and prognostic ability of changes in indices of cardiac mechanics (strain, ESPVR and VA coupling) via serial echocardiographic assessment in a prospective, multicentre community-based cohort of adults treated with anthracycline chemotherapy.

\section{METHODS}

\section{Study population}

The PREDICT study, A Multicenter Study in Patients Undergoing AnthRacycline-Based Chemotherapy to Assess the Effectiveness of Using Biomarkers to Detect and Identify Cardiotoxicity and Describe Treatment (CinicalTrials.gov Identifier: NCT01032278), was an National Cancer Institute (NCI)-funded, multicentre, longitudinal cohort study of participants undergoing anthracycline-based chemotherapy across 24 US community oncology programmes with serial assessments of cardiac function via cardiac imaging. The primary inclusion criteria were patients aged 18-85 years with life expectancy $>12$ months who were initiating a course of anthracycline-based chemotherapy. Exclusion criteria for the parent study were as follows: unstable angina, myocardial infarction, decompensated HF or symptomatic arrhythmia within 3 months of registration; $\mathrm{EF}<50 \%$ as determined by each clinical site via visual estimation; concurrent dexrazoxane exposure; severe pulmonary disease (forced expiratory volume $\leq 1.0 \mathrm{~L}$ ); pulmonary hypertension (mean pulmonary artery pressure $\geq 60 \mathrm{~mm} \mathrm{Hg}$ ); oxygen dependence; B-type natriuretic peptide $\geq 200 \mathrm{pg} / \mathrm{mL}$ or troponin $\geq 0.4 \mathrm{ng} / \mathrm{mL}$.

As specified by the trial protocol, echocardiography and clinical assessments were performed on participants at baseline prior to the initiation of chemotherapy, 6 months, 12 months and at additional time points if clinically indicated.

All participants provided written, informed consent, and the echocardiography substudy protocol was approved by the Institutional Review Board at each participating site.

\section{Quantitative echocardiography analyses}

Echocardiograms were performed at each community oncology site by clinical sonographers according to standard institutional protocol. Echocardiograms were acquired primarily on the GE, Philips and Sequoia platforms. For the echocardiography substudy, sites were contacted after trial completion with requests for deidentified echocardiographic DICOM images. These archived images obtained post hoc from sites were stored at the acquisition frame rate in $\sim 40 \%$ of studies and compressed to 30 frames per second in $\sim 60 \%$ of studies. Participants with a baseline and at least 1 follow-up echocardiogram were included in these echocardiography subcohort analyses.

Echocardiograms were subsequently analysed at the University of Pennsylvania Center for Quantitative Echocardiography (Philadelphia, Pennsylvania, USA) with sonographers blinded to all clinical characteristics. The following measures were quantified: EF; longitudinal, circumferential and radial strain and strain rate; and Doppler timing interval measures to derive the ESPVR and VA interaction indices $\mathrm{Ees}_{\mathrm{sb}}, \mathrm{V}_{100}$, Ea and $\mathrm{Ea} / \mathrm{Ees}_{\mathrm{sb}}$. All measurements were performed using Tomtec 2D Cardiac Performance Analysis (TomTec Imaging Systems, Unterschleissheim, Germany). The quantitation and derivation of these parameters have been previously described and are further summarised below. ${ }^{9} 1113$

Apical 4-chamber LV end-diastolic volumes (EDV) and end-systolic volumes (ESV) were determined using the Simpson's method of discs and used to derive stroke volume (SV) and EF, as recommended by the American Society of Echocardiography. ${ }^{14}$ To determine longitudinal, circumferential and radial strain and strain rate, the LV endocardial border was manually traced at the end-systolic frame of one cardiac cycle from the apical 
4-chamber and the mid-papillary level short-axis views. Tracings with poor tracking were adjusted in individual segments. Peak systolic strain and strain rate values were computed and averaged across segments. We used the following criteria to exclude images from analyses secondary to inadequate quality: (1) significant foreshortening of the apical view, that is, when the maximum length of the LV was not displayed or when the apex was out of sector; (2) lack of visualisation of the LV endocardium due to under-gaining or artefact; (3) oblique short-axis views rather than circular, that is, when the $\mathrm{LV}$ minor axes were too dissimilar. Image quality was inadequate in the apical 2-chamber view in $\sim 25 \%$ of echocardiograms; thus, EF and longitudinal strain were derived from the 4-chamber view in order to ensure consistency. Of note, prior studies from our own group and others have demonstrated a strong correlation between longitudinal strain obtained from the apical 4-chamber view and the apical 2-chamber view, in addition to adequate reproducibility of longitudinal strain derived from the 4-chamber view alone. ${ }^{11} 1315$

In order to derive measures of the ESPVR and VA interaction, the end-systolic elastance $\left(\mathrm{Ees}_{\mathrm{sb}}\right)$ was determined using a modified single-beat algorithm described and validated by Chen et al. ${ }^{13} 16$ This algorithm includes timing intervals measured in standardly acquired Doppler interrogation of the LV outflow tract and LV volumes derived via the Simpson's method. $\mathrm{V}_{100}$, the theoretical LV volume at an end-systolic pressure (ESP) of $100 \mathrm{~mm} \mathrm{Hg}$, was determined using the $\mathrm{Ees}_{\mathrm{sb}}$, ESV and the ESP. ${ }^{9}$ Effective arterial elastance $(\mathrm{Ea})$ was calculated to be (ESP)/SV, where ESP was estimated as $0.9 \times$ systolic pressure measured by brachial blood pressure. ${ }^{16}$ The VA coupling index is determined by the ratio of $\mathrm{Ea} / \mathrm{Ees}_{\mathrm{sb}}$. For blood pressure recordings, a single measurement was performed as per each institutional protocol.

For all studies, LV volumes and strain were quantified by a single research sonographer. Doppler timing intervals for the calculation of $\mathrm{Ees}_{\mathrm{sb}}$ and $\mathrm{V}_{100}$ were measured by a separate research sonographer. Both are highly experienced in echocardiographic quantitation. Reproducibility analyses $(n=25)$ demonstrated intraobserver coefficients of variation of $2.5-3.9 \%$ for $\mathrm{LV}$ volumes, $3.2 \%$ for $\mathrm{EF}, 9.1 \%$ for longitudinal strain, $7.9 \%$ for circumferential strain and $34.9 \%$ for radial strain. For Doppler timing intervals, intraobserver coefficients of variation were 1.1-6.3\%.

\section{Statistical methods}

Standard descriptive statistics were used to summarise participant characteristics and baseline echocardiographic measurements. Fisher's exact and Wilcoxon rank sum tests were used to compare characteristics of participants from the parent study who were included and excluded from this echocardiography substudy based on the availability of analysable echocardiograms. We defined the outcome of CTRCD as an EF decrease $\geq 10 \%$ from baseline to $<50 \%$ as assessed in the echocardiographic core laboratory. These criteria were based on prior studies in oncology and cardio-oncology. ${ }^{17} \quad 18$ Given that CTRCD is defined by a change in EF from baseline, participants were only at risk for CTCRD at follow-up visits; they could not have CTRCD at the baseline visit.

We created two series of statistical models: 'diagnostic' and 'prognostic'. First, we determined the associations of measures with the outcome CTRCD at the same follow-up visit. These analyses were considered 'diagnostic' models. All echocardiograms from all participants in this subcohort study were included in these models. In order to account for the longitudinal nature of the data, logistic regression models using the generalised estimating equation method were used. An exchangeable correlation structure was specified and a robust variance estimator was used. In these models, changes in each echocardiography measure and the outcome of CTRCD were assessed at the same follow-up visit. Each model was adjusted for age, sex and the baseline echocardiography value for the parameter of interest.

Next, we considered 'prognostic' models. We determined the associations between changes in each echocardiography measure from baseline to 6 months, and the outcome CTCRD at 12 months. Only participants who had three quantifiable echocardiograms at the baseline, 6 and 12-month time points were included in these analyses. Fisher's exact and Wilcoxon rank sum tests were used to compare baseline characteristics and echocardiography measures of participants within the echocardiography substudy who were included in and excluded from these analyses based on the availability of analysable echocardiograms. We used logistic regression models with the outcome CTCRD at 12 months, and the predictor being change in each measure at 6 months, adjusted for age, sex and baseline value. In sensitivity analyses, we assessed for potential confounding by anthracycline dose. Furthermore, we tested for multicollinearity by examining the CIs and variance inflation factors in each model. The linearity assumption was also assessed through graphical depiction of the Pearson residuals of these models in relation to the changes in measures at 6 months. For each measure in which the change at 6 months demonstrated significant associations with CTRCD at 12 months, receiver operating characteristic (ROC) curves were constructed, and the area under the curve (AUC) was determined.

All tests were two-sided and $\mathrm{p}$ values of 0.05 or less were considered statistically significant. Statistical analyses were carried out using SAS V.9 (SAS Institute, Cary, North Carolina, USA).

\section{RESULTS}

\section{Echocardiography subcohort population}

Of the 598 participants in the parent PREDICT trial, 165 participants contributed 493 echocardiograms to the echocardiography subcohort study. As compared to the parent study, participants from the echocardiography subcohort were more likely to be of white race, to have hyperlipidaemia and to have a slightly lower body mass index (BMI), 
while other demographic and clinical characteristics were similar (see online supplementary table $\mathrm{S} 1$ ).

Participant characteristics of this echocardiography subcohort are summarised in table 1 . The mean age was 53 years. The majority of participants were Caucasian and female, and $\sim 61 \%$ of participants had breast cancer.

Table 1 Characteristics of the study participants

\begin{tabular}{|c|c|}
\hline & $\begin{array}{l}\text { All participant } \\
\qquad(\mathrm{n}=165)\end{array}$ \\
\hline \multicolumn{2}{|l|}{ Demographic characteristics } \\
\hline Age, years (SD) & $53(13)$ \\
\hline Female sex, $\mathrm{n}(\%)$ & $127(77)$ \\
\hline \multicolumn{2}{|l|}{ Race, n (\%) } \\
\hline White & $149(90)$ \\
\hline Black & $14(8)$ \\
\hline Other & $2(2)$ \\
\hline \multicolumn{2}{|l|}{ Cancer and related therapies } \\
\hline \multicolumn{2}{|l|}{ Cancer type, n (\%) } \\
\hline Breast cancer & $101(61)$ \\
\hline Lymphoma & $48(29)$ \\
\hline Leukaemia & $9(6)$ \\
\hline Sarcoma & $7(4)$ \\
\hline \multicolumn{2}{|l|}{ Anthracycline agent, $\mathrm{n}(\%)$} \\
\hline Doxorubicin & $149(90)$ \\
\hline Other anthracycline agent & $16(10)$ \\
\hline Anthracycline dose, ${ }^{\star} \mathrm{mg} / \mathrm{m}^{2}$ (SD) & $240(60)$ \\
\hline Trastuzumab, n (\%) & $13(8)$ \\
\hline Chest radiation therapy, $\mathrm{n}(\%)$ & $1(<1)$ \\
\hline $\begin{array}{l}\text { Prior anthracycline or chest radiation } \\
\text { exposure, } n(\%)\end{array}$ & $7(4)$ \\
\hline \multicolumn{2}{|l|}{ Medical history and risk factors } \\
\hline Diabetes, n (\%) & $18(11)$ \\
\hline Hypertension, n (\%) & 52 (32) \\
\hline Hypothyroidism, n (\%) & $2(1)$ \\
\hline Hyperlipidaemia, n (\%) & $33(20)$ \\
\hline $\begin{array}{l}\text { Number of cardiovascular risk } \\
\text { factorst, median (IQR) }\end{array}$ & $2(1-3)$ \\
\hline Prior cardiomyopathy, n (\%) & $3(2)$ \\
\hline Current or prior tobacco use, $\mathrm{n}(\%)$ & $70(42)$ \\
\hline Body mass index, $\mathrm{kg} / \mathrm{m}^{2}(\mathrm{SD})$ & $28.4(6.2)$ \\
\hline Systolic blood pressure, mm Hg (SD) & $126(16)$ \\
\hline Diastolic blood pressure, $\mathrm{mm} \mathrm{Hg}$ (SD) & $73(10)$ \\
\hline Heart rate, beats/min (SD) & 74 (13) \\
\hline \multicolumn{2}{|l|}{ Cardiac medications, $\mathrm{n}(\%)$} \\
\hline Any & $69(42)$ \\
\hline $\begin{array}{l}\text { ACE inhibitor or angiotensin } \\
\text { receptor blocker }\end{array}$ & $23(14)$ \\
\hline$\beta$ blocker & $38(23)$ \\
\hline HMG-CoA reductase inhibitor & $30(18)$ \\
\hline Aspirin & $21(13)$ \\
\hline
\end{tabular}

${ }^{*}$ Anthracycline dose is given in doxorubicin equivalents, with doxorubicin $1 \mathrm{mg} / \mathrm{m}^{2}$ being considered equivalent to daunorubicin $1.0 \mathrm{mg} / \mathrm{m}^{2}$, epirubicin $0.67 \mathrm{mg} / \mathrm{m}^{2}$, idarubicin $3.0 \mathrm{mg} / \mathrm{m}^{2}$ and mitoxantrone $4.0 \mathrm{mg} / \mathrm{m}^{2} .^{19}$

†Risk factors include history of myocardial infarction, coronary artery disease, hypertension, alcohol abuse, substance abuse cardiomyopathy, diabetes mellitus, obesity, smoking, hypothyroidism, hyperlipidaemia, anthracycline exposure, trastuzumab exposure or radiation therapy; family history of heart disease; concurrent radiation therapy and age older than 65 years. HMG-CoA, 3-hydroxy-3-methylglutaryl coenzyme A.
Approximately $90 \%$ of the participants received doxorubicin, with the remainder receiving daunorubicin, epirubicin, idarubicin or mitoxantrone. Using published anthracycline chemotherapy dose conversions, ${ }^{19}$ the median dose of anthracycline was equivalent to $240 \mathrm{mg}$ / $\mathrm{m}^{2}$ of doxorubicin. Although four participants $(2 \%)$ had a history of intermittent atrial fibrillation, all were in sinus rhythm at the time of their echocardiograms. The cohort was of high cardiovascular risk, with $101(61 \%)$ participants having at least two risk factors, a mean BMI of $28.4 \mathrm{~kg} / \mathrm{m}^{2}$, and $42 \%$ treated with cardiac medications.

\section{Echocardiographic measures and outcomes}

At baseline, the mean EF, longitudinal strain, circumferential strain and radial strain were $50.0 \%,-14.9 \%$, $-23.6 \%$ and $45.8 \%$, respectively (table 2 ). The mean values for $\mathrm{Ees}_{\mathrm{sb}}, \mathrm{V}_{100}, \mathrm{Ea}$ and $\mathrm{Ea} / \mathrm{Ees}_{\mathrm{sb}}$ were $2.1 \mathrm{~mm} \mathrm{Hg}$ / $\mathrm{mL}, 53.9 \mathrm{~mL}, 2.0 \mathrm{~mm} \mathrm{Hg} / \mathrm{mL}$ and 1.0 unit, respectively. These baseline measures are notable for borderline normal values in multiple indices, including EF and longitudinal strain, in comparison to normal ranges for our laboratory and other published reference ranges. ${ }^{14} 2021$

Thirty-one participants (19\%) developed CTRCD during the study period, as defined by a decline in EF by $\geq 10 \%$ from baseline to $<50 \%$ as measured by the core laboratory. Two participants with CTCRD developed symptomatic $\mathrm{HF}$ as determined by the treating clinician during the study period, one within 6 months and one at the time of the 6-month echocardiogram. In addition, of the 31 participants with CTRCD, $15(48 \%)$ reported oedema, $27(87 \%)$ reported fatigue and $6(19 \%)$ reported paroxysmal nocturnal dyspnoea. While four participants died of cancer, none died of cardiovascular causes during the study period.

\section{Associations between changes in mechanics measures and CTRCD at the same follow-Up visit}

We determined the associations between changes in values of mechanics measures with the outcome of CTRCD assessed at the same follow-up visit using all echocardiograms from all 165 participants (table 3). The majority of the echocardiograms $(\sim 90 \%)$ in these

Table 2 Echocardiographic parameters at baseline

\begin{tabular}{lc}
\hline & $\begin{array}{c}\text { All participants } \\
(\mathbf{n}=\mathbf{1 6 5 )}\end{array}$ \\
\hline Ejection fraction, \% (SD) & $50.0(5.8)$ \\
Longitudinal strain, \% (SD) & $-14.9(2.8)$ \\
Circumferential strain, \% (SD) & $-23.6(5.4)$ \\
Radial strain, \% (SD) & $45.8(22.8)$ \\
Longitudinal strain rate, 0.1/s (SD) & $-0.9(0.2)$ \\
Circumferential strain rate, 0.1/s (SD) & $-1.5(0.5)$ \\
Radial strain rate, 0.1/s (SD) & $2.3(1.0)$ \\
Ees $_{\text {sb }} 0.1 \mathrm{~mm} \mathrm{Hg} / \mathrm{mL}(\mathrm{SD})$ & $2.1(0.7)$ \\
$\mathrm{V}_{100}, \mathrm{~mL}(\mathrm{SD})$ & $53.9(15.5)$ \\
Ea, $0.1 \mathrm{~mm} \mathrm{Hg} / \mathrm{mL}(\mathrm{SD})^{\text {Ea/Ees }} \mathrm{sb}_{\mathrm{sb}}, 0.1 \mathrm{unit}(\mathrm{SD})$ & $2.0(0.5)$ \\
& $1.0(0.3)$ \\
\hline
\end{tabular}


analyses occurred at baseline, 6 or 12 months, while the remaining $10 \%$ occurred at other time points at the discretion of the clinical provider (eg, monitoring during trastuzumab therapy). The median time that follow-up echocardiograms were obtained for these 'diagnostic' analyses was 7 months (IQR 6-12). Changes in longitudinal and circumferential strain and strain rate and changes in $\mathrm{V}_{100}$, Ea and $\mathrm{Ea} / \mathrm{Ees}_{\mathrm{sb}}$ each demonstrated

Table 3 Associations of baseline values and changes in measures of mechanics with CTRCD at the same follow-up visit $(n=165)$

\begin{tabular}{|c|c|c|}
\hline $\begin{array}{l}\text { Echocardiographic } \\
\text { measure }\end{array}$ & $\begin{array}{c}\text { Adjusted OR* } \\
(95 \% \mathrm{Cl})\end{array}$ & p Value \\
\hline \multicolumn{3}{|l|}{ Strain } \\
\hline \multicolumn{3}{|c|}{ Longitudinal strain, \% } \\
\hline Baseline† & $1.01(0.995$ to 1.03$)$ & 0.182 \\
\hline Change & 1.02 (1.01 to 1.03$)$ & 0.004 \\
\hline \multicolumn{3}{|c|}{ Circumferential strain, \% } \\
\hline Baseline† & $1.00(0.993$ to 1.01$)$ & 0.526 \\
\hline Change & 1.01 (1.00 to 1.02$)$ & 0.009 \\
\hline \multicolumn{3}{|l|}{ Radial strain, \% } \\
\hline Baseline† & $0.999(0.996$ to 1.00$)$ & 0.413 \\
\hline Change & 0.999 (0.998 to 1.00$)$ & 0.241 \\
\hline \multicolumn{3}{|l|}{ Strain rate } \\
\hline \multicolumn{3}{|c|}{ Longitudinal strain rate, $0.1 / \mathrm{s}$} \\
\hline Baseline§ & $1.01(0.994$ to 1.03$)$ & 0.201 \\
\hline Changeף & $1.03(1.01$ to 1.04$)$ & 0.003 \\
\hline \multicolumn{3}{|c|}{ Circumferential strain rate, $0.1 / \mathrm{s}$} \\
\hline Baseline§ & $1.01(0.999$ to 1.02$)$ & 0.089 \\
\hline Changeף & 1.01 (1.00 to 1.02$)$ & 0.018 \\
\hline \multicolumn{3}{|c|}{ Radial strain rate, $0.1 / \mathrm{s}$} \\
\hline Baseline§ & 0.998 (0.994 to 1.00$)$ & 0.422 \\
\hline Changeף & 0.999 (0.996 to 1.00$)$ & 0.420 \\
\hline \multicolumn{3}{|c|}{ ESPVR and VA coupling } \\
\hline \multicolumn{3}{|c|}{$\mathrm{Ees}_{\mathrm{sb}}, 0.1 \mathrm{~mm} \mathrm{Hg} / \mathrm{mL}$} \\
\hline Baseline $^{\star \star}$ & $1.01(0.995$ to 1.02$)$ & 0.272 \\
\hline Change†† & 1.01 (0.999 to 1.02$)$ & 0.068 \\
\hline \multicolumn{3}{|l|}{$\mathrm{V}_{100}, 10 \mathrm{~mL}$} \\
\hline Baseline $\ddagger$ & 0.993 (0.957 1.03) & 0.703 \\
\hline Change§§ & 1.07 (1.04 to 1.10$)$ & $<0.001$ \\
\hline \multicolumn{3}{|l|}{$\mathrm{Ea}, 0.1 \mathrm{~mm} \mathrm{Hg} / \mathrm{mL}$} \\
\hline Baseline ${ }^{\star \star}$ & $1.01(0.996$ to 1.02$)$ & 0.192 \\
\hline Change†† & 1.03 (1.02 to 1.04$)$ & $<0.001$ \\
\hline \multicolumn{3}{|l|}{$\mathrm{Ea} / \mathrm{Ees}_{\mathrm{sb}}, 0.1$ unit } \\
\hline Baselineףी & 0.998 (0.976 to 1.02$)$ & 0.847 \\
\hline Change $e^{\star \star \star}$ & $1.02(1.00$ to 1.04$)$ & 0.034 \\
\hline
\end{tabular}

${ }^{\star}$ Adjusted for age and sex; baseline values and changes in measures are included in each model and their respective associations with CTCRD at follow-up visits are reported in the table.

TOR of CTRCD for every $1 \%$ difference at baseline. ¥OR of CTRCD for every $1 \%$ increase from baseline. $\S O R$ of CTRCD for every $0.1 / \mathrm{s}$ difference at baseline. TOR of CTRCD for every $0.1 / \mathrm{s}$ increase from baseline. ${ }^{* *}$ OR of CTRCD for every $0.1 \mathrm{~mm} \mathrm{Hg} / \mathrm{mL}$ difference at baseline. ††OR of CTRCD for every $0.1 \mathrm{~mm} \mathrm{Hg} / \mathrm{mL}$ increase from baseline. $\ddagger \ddagger O R$ of CTRCD for every $10 \mathrm{~mL}$ difference at baseline. $\S \S \mathrm{OR}$ of CTRCD for every $10 \mathrm{~mL}$ increase from baseline. TाTOR of CTRCD for every 0.1 unit difference at baseline. ${ }^{\star \star \star}$ OR of CTRCD for every 0.1 unit increase from baseline. CTRCD, Cancer Therapeutics-Related Cardiac Dysfunction; ESPVR, end-systolic pressure-volume relationship. significant associations with CTCRD. In models adjusted for age, sex and baseline measure, the overall effect size was modest. A $1 \%$ worsening of longitudinal or circumferential strain, a $0.1 / \mathrm{s}$ worsening of longitudinal or circumferential strain rate, a $0.1 \mathrm{~mm} \mathrm{Hg} / \mathrm{mL}$ increase in $\mathrm{Ea}$ and a 0.1 unit increase in $\mathrm{Ea} / \mathrm{Ees}_{\mathrm{sb}}$ were each

Table 4 Associations between echocardiographic measures at baseline and changes at 6 months with CTRCD at 12 months $(n=103)$

\begin{tabular}{lcl}
\hline $\begin{array}{l}\text { Echocardiographic } \\
\text { measure }\end{array}$ & $\begin{array}{c}\text { Adjusted OR* } \\
(95 \% \mathrm{CI})\end{array}$ & p Value \\
\hline
\end{tabular}

\section{Strain}

Longitudinal strain, \%

$\begin{array}{lll}\text { Baseline } \dagger & 1.09(0.856 \text { to } 1.38) & 0.493 \\ \text { Change } & 1.18(0.949 \text { to } 1.47) & 0.137\end{array}$

Circumferential strain, \%

Baseline† $\quad 0.883(0.743$ to 1.05$) \quad 0.159$

Change

$0.862(0.731$ to 1.02$) \quad 0.079$

Radial strain, \%
Baseline†
$1.00(0.967$ to 1.03$) \quad 0.978$

Change

1.00 (0.978 to 1.02$) \quad 0.973$

\section{Strain rate}

Longitudinal strain rate, $0.1 / \mathrm{s}$

$\begin{array}{lll}\text { Baseline } & 1.17(0.843 \text { to } 1.62) & 0.350 \\ \text { Changeף } & 1.51(1.08 \text { to } 2.12) & 0.016\end{array}$

Circumferential strain rate, $0.1 / \mathrm{s}$

$\begin{array}{lrl}\text { Baseline } & 1.01(0.844 \text { to } 1.22) & 0.882 \\ \text { Changeq } & 0.985(0.859 \text { to } 1.13) & 0.834\end{array}$

Radial strain rate, $0.1 / \mathrm{s}$

Baseline§ $\quad 0.985$ (0.911 to 1.07$) \quad 0.711$

Changeף

0.993 (0.940 to 1.05$) \quad 0.811$

\section{ESPVR and VA coupling}

Ees $_{\mathrm{sb}}, 0.1 \mathrm{~mm} \mathrm{Hg} / \mathrm{mL}$

Baseline**

Change†t

0.958 (0.848 to 1.08$) \quad 0.490$

$\mathrm{V}_{100}, 10 \mathrm{~mL}$

Baseline $\ddagger$

Change§§

0.997 (0.907 to 1.10$) \quad 0.954$

$\mathrm{Ea}, 0.1 \mathrm{~mm} \mathrm{Hg} / \mathrm{mL}$

Baseline**

Change†t

$\mathrm{Ea} / \mathrm{Ees}_{\mathrm{sb}}, 0.1$ unit

Baselineๆ

Change ${ }^{\star \star \star}$

0.661 (0.393 to 1.11$) \quad 0.120$

2.13 (1.33 to 3.43$) \quad 0.002$

1.06 (0.916 to 1.23$) \quad 0.424$

1.19 (1.04 to 1.36$) \quad 0.010$

1.12 (0.881 to 1.42$) \quad 0.357$

$1.12(0.965$ to 1.31$) \quad 0.132$

${ }^{*}$ Adjusted for age and sex.

TOR of CTRCD for every $1 \%$ difference at baseline.

$\neq$ OR of CTRCD for every $1 \%$ increase from baseline to 6 months.

$\S$ ○R of CTRCD for every $0.1 / \mathrm{s}$ difference at baseline.

ПOR of CTRCD for every $0.1 / \mathrm{s}$ increase from baseline to

6 months.

${ }^{* *}$ OR of CTRCD for every $0.1 \mathrm{~mm} \mathrm{Hg} / \mathrm{mL}$ difference at baseline.

††OR of CTRCD for every $0.1 \mathrm{~mm} \mathrm{Hg} / \mathrm{mL}$ increase from baseline to 6 months.

¥¥OR of CTRCD for every $10 \mathrm{~mL}$ difference at baseline.

$\S \S \mathrm{OR}$ of CTRCD for every $10 \mathrm{~mL}$ increase from baseline to 6 months.

११OR of CTRCD for every 0.1 unit difference at baseline.

${ }^{* \star *}$ OR of CTRCD for every 0.1 unit increase from baseline to 6 months.

CTRCD, Cancer Therapeutics-Related Cardiac Dysfunction; ESPVR, end-systolic pressure-volume relationship. 
associated with a 1-3\% increased odds of CTCRD (all $\mathrm{p}<0.05)$. A $10 \mathrm{~mL}$ increase in $\mathrm{V}_{100}$, indicative of worse cardiac remodelling, was associated with a $7 \%$ increase in the odds of CTRCD $(\mathrm{p}<0.001)$.

\section{Associations between changes in mechanics measures at 6 months and CTRCD at 12 months}

There were 103 participants with analysable echocardiograms at baseline, 6 and 12 months and were thus included in the 'prognostic' analyses. There were no significant differences in the baseline characteristics or echocardiography measures between those participants included in and excluded from these analyses (see online supplementary table S2). Of these 103 participants, $19(18 \%)$ had the outcome of CTRCD at 12 months. In these analyses, we determined the prognostic significance of changes in mechanics measures by defining the associations between changes in measures from baseline to 6 months with CTCRD at 12 months, again adjusted for age, sex and baseline measures (table 4). Here, longitudinal strain rate, $\mathrm{V}_{100}$ and $\mathrm{Ea}$ were each significantly associated with CTRCD. A $0.1 / \mathrm{s}$ worsening of longitudinal strain rate (OR 1.51 (95\% CI 1.08 to 2.12 )), $10 \mathrm{~mL}$ increase in $\mathrm{V}_{100}$ (OR 2.13 (95\% CI 1.33 to 3.43 ) and a $0.1 \mathrm{~mm} \mathrm{Hg} / \mathrm{mL}$ increase in $\mathrm{Ea}$ (OR 1.19 (95\% CI 1.04 to 1.36 )) were individually and strongly associated with CTCRD at 12 months. ROC curves derived from these adjusted models for longitudinal strain rate (AUC 0.719 (95\% CI 0.595 to 0.843 )), $\mathrm{V}_{100}$ (AUC 0.796 (95\% CI 0.689 to 0.903 ) ) and Ea (AUC 0.742 (95\% CI 0.632 to 0.852$)$ ) each demonstrated predictive utility (figure 1 ).

We performed sensitivity analyses incorporating additional adjustment for anthracycline dose, a covariate that demonstrated an unadjusted OR for CTRCD of 1.07 ( $95 \%$ CI 0.999 to $1.15, \mathrm{p}=0.053$ ). The associations of baseline values and changes at 6 months in each measure with CTRCD at 12 months after adjusting for age, sex and anthracycline dose were consistent with our original findings, with 6-month changes in longitudinal strain rate $(\mathrm{p}=0.02)$, Ea $(\mathrm{p}=0.009)$ and $\mathrm{V}_{100}(\mathrm{p}=0.002)$ being the only measures associated with CTRCD at 12 months.

\section{DISCUSSION}

In this prospective, multicentre study of anthracyclinetreated adults from community-based oncology centres, we determined the following: (1) changes in longitudinal and circumferential strain and strain rate, $\mathrm{V}_{100}$, Ea and $\mathrm{Ea} / \mathrm{Ees}_{\mathrm{sb}}$ are each associated with CTRCD at the same visit; and (2) changes in longitudinal strain rate, $\mathrm{Ea}$ and $\mathrm{V}_{100}$ from baseline to 6 months are strongly predictive of CTRCD at 12 months. While a number of prior investigations in tertiary care centres have suggested a role for myocardial mechanics in cardio-oncology, ${ }^{13}$ 22-25 our data provide external validity to the relevance of non-invasively derived measures of myocardial mechanics to community-based populations

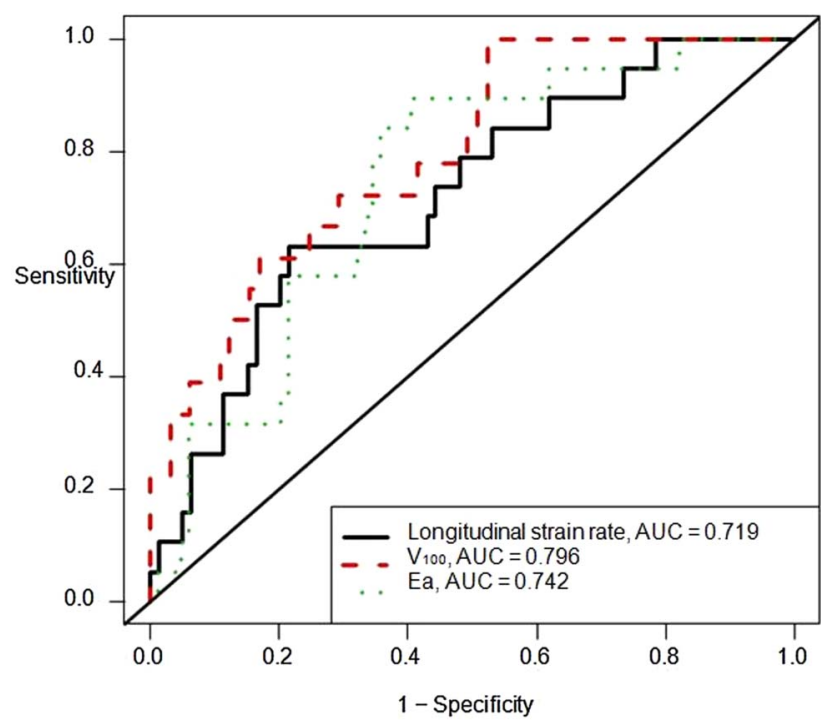

Figure 1 Receiver operating characteristics (ROC) curves demonstrating the predictive ability of echocardiographic measures. ROC curves and the area under the curve (AUC) for the prediction of cancer therapeutics-related cardiac dysfunction (CTRCD) at 12 months according to the changes in measures of mechanics at 6 months. All models were adjusted for baseline echocardiography measure, age and sex. Depicted measures include longitudinal strain rate (black); $\mathrm{V}_{100}$ (red); Ea (green).

treated with anthracyclines. Moreover, our findings emphasise the critical importance of assessing change over time in imaging measures with cancer therapy.

There has been limited prior study of cardiac mechanics, particularly non-invasively derived indices of cardiac contractility, arterial load and VA coupling, in cohorts of patients exposed to cancer therapies. Our prior data in a tertiary-referral breast cancer population first suggested that $\mathrm{Ea}, \mathrm{Ea} / \mathrm{Ees}_{\mathrm{sb}}$ as well as longitudinal and circumferential strain and strain rate hold important diagnostic and prognostic relevance. ${ }^{13}$ This current study suggests that these indices are also valid in community-based populations. In addition, we expand on our prior findings by highlighting the prognostic importance of $\mathrm{V}_{100}$, a measure of the ESPVR that has not previously been studied in cardio-oncology. $\mathrm{V}_{100}$ provides insight into LV remodelling and contractility, as it represents the intercept of the line describing the ESPVR of each cardiac cycle. Prior studies in mild and severe HF support the importance of this measure, ${ }^{9} 1126$ and we postulate that increases in $\mathrm{V}_{100}$ likely represent the progressive LV dilation and cardiac remodelling that occurs in response to these agents. Further work is needed to validate the predictive utility of $\mathrm{V}_{100}$. Moreover, this current study provides evidence to support our prior findings that changes in arterial load (Ea) are reproducibly associated with CTRCD and prognosis in $\mathrm{HF}^{9}{ }^{13}$ Specifically focusing on modifying afterload may be of benefit and a readily modifiable target in patients exposed to these cardiotoxic cancer therapies. 
Our study highlights the critical need for more detailed characterisation of arterial properties and their impact on outcomes in cardio-oncology.

Similar to our previous work and the work of others, we determined the predictive utility of longitudinal strain rate. ${ }^{132427}$ We also found diagnostic associations between longitudinal and circumferential strain and cardiac dysfunction. ${ }^{13}$ However, we did not find changes in longitudinal or circumferential strain to be predictive of subsequent outcomes. ${ }^{132324}$ Although initially counterintuitive, there are a number of potential reasons for this finding, including differences in image acquisition, strain software or tracing methodology across studies; variations in study design, analytic methods and participant characteristics across studies; and sample size limitations. It is also plausible that measurement error played a role, although our reproducibility analyses suggest very low variability.

There may, however, also be biological factors that explain these findings, particularly with regard to circumferential strain. Longitudinal, circumferential and radial strain measurements each reflect different dimensions of myocardial contractility and contribute to the global measure of EF. When myocardial injury occurs, dysfunction is typically not uniform across these dimensions. Studies performed in a variety of HF populations have suggested that longitudinal deformation is the first dimension of myocardial strain to become impaired,${ }^{10}{ }^{28}$ potentially reflecting vulnerability of the subendocardium. ${ }^{29}$ In this setting, circumferential strain may initially play a compensatory role in response to early cardiac injury, thereby resulting in preservation of EF. ${ }^{31}$ However, with further cardiac injury, the response may be deterioration of circumferential strain accompanied by reduced $\mathrm{EF}^{28}{ }^{32}$ It may be that temporal factors dictate the predictive relevance of circumferential strain. More specifically, it may be that circumferential strain is predictive in the longer, but not shorter term. In this study, follow-up was only 12 months, whereas in our prior study, participants were followed for up to 3 years.

Phase III clinical trial data, in which patients with significant cardiovascular conditions are typically excluded, suggest that $15-17 \%$ of participants exposed to anthracyclines experience declines in left ventricular ejection fraction (LVEF) of $>20 \%$ and the majority of these are less than the limit of normal. ${ }^{3}$ In our study, there was a similarly high rate of CTRCD (19\%), and we postulate that this is likely to be due to factors specific to the study participants. First, the baseline EF and longitudinal strain are at the lower limit of normal, in comparison to both published normal ranges, ${ }^{14} 20$ and our laboratory reference ranges. The substantial prevalence of cardiovascular risk factors, in addition to the low normal function as assessed by echocardiographic measures, suggests that this is a high cardiovascular risk population. These cardiovascular risk factors likely contributed to a greater frequency of CTRCD and may affect the generalisability, but not the validity, of our results.

The authors acknowledge the potential limitations of this study. First, echocardiography studies were acquired on multiple platforms using institution-specific protocols. Potential sources of variability with strain imaging have been well described, ${ }^{33}$ and the variability introduced by different acquisition platforms and compressed frame rates in some studies may have affected our ability to detect significant findings. Prospective implementation of standard imaging protocols is of critical importance, and this would likely have enhanced the quality of data collection and analysability of images. Images from the apical 2-chamber were particularly limited, and our findings with respect to longitudinal strain may be impacted by the use of a single view for assessment, though a recent intervendor study found that longitudinal strain derived from the 4-chamber view alone was reproducible and strongly correlated with values derived from all three apical views. ${ }^{15}$ For these reasons, we refrain from making definitive comparisons of the relative utility of different measures in the prediction of CTCRD. Despite this limitation, our study provides evidence to support the relevance of these measures and the feasibility of their quantitation, even in a post hoc manner. Second, our radial strain values were highly variable, suggesting that this measure may be of somewhat lesser utility, particularly when obtained retrospectively. Third, our sample size was limited given lack of availability of echocardiograms for many parent study participants, although participant characteristics between the parent study and our subcohort were largely similar. Additionally, a subset of participants could not be included in the 'prognostic' analyses due to lack of availability of echocardiograms at the 6-month or 12-month time point. The sample size limitation precluded our assessment of the impact of cardiac medications on the prediction of CTRCD and our ability to compare the prognostic associations of multiple echocardiography measures in the same model. However, these missing data are unlikely to affect the validity of our findings given the largely similar characteristics between included and excluded participants. Finally, type 1 error is a possibility as multiple comparisons were performed, though it is unlikely that the number of significant associations could have been attributable to chance alone. Overall, these limitations highlight the critical importance of prospective standardisation of echocardiography acquisition and archiving in clinical studies.

In conclusion, we demonstrate that changes in measures of longitudinal strain rate, the ESPVR and arterial load are each associated with and predictive of cardiac dysfunction after anthracycline therapy in this community-based cohort. With further study, these data suggest the potential utility of these measures broadly in clinical practice.

\section{Author affiliations}

${ }^{1}$ Division of Cardiology, Department of Pediatrics, The Children's Hospital of Philadelphia, Philadelphia, Pennsylvania, USA

${ }^{2}$ Department of Biostatistics, The University of Texas MD Anderson Cancer Center, Houston, Texas, USA 
${ }^{3}$ Department of Medicine, Vanderbilt University Medical Center, Nashville, Tennessee, USA

${ }^{4}$ Department of Medicine, Division of Cardiovascular Medicine, The University of Pennsylvania, Philadelphia, Pennsylvania, USA

${ }^{5}$ Department of General Oncology, The University of Texas MD Anderson Cancer Center, Houston, Texas, USA

${ }^{6}$ Department of Biostatistics and Epidemiology, The University of Pennsylvania, Philadelphia, Pennsylvania, USA

Contributors BK designed the echocardiography study, monitored data collection, wrote the statistical analysis plan, analysed the data and drafted and revised the manuscript. She is guarantor. HKN wrote the statistical analysis plan, analysed the data and drafted and revised the manuscript. WW and ZF analysed the data and drafted and revised the manuscript. TP and VE performed data collection and analyses of all echocardiograms and critically reviewed and revised the manuscript. DL and MF conceived the parent study, implemented the trial, monitored data collection and critically reviewed and revised the manuscript. All authors provided final approval of the manuscript.

Funding This study was supported by the NCI U10 CA 189828-02, U10 CA 045809-25, and an NCl Echocardiography Supplement. BK was supported by NHLBI R01-HL118018 and NHLBI K23-HL095661. HKN was supported by NICHD T32-HD060550. WW was supported by NIH P30 CA016672.

Competing interests None declared.

Ethics approval Institutional Review Board of each participating centre.

Provenance and peer review Not commissioned; externally peer reviewed.

Data sharing statement No additional data are available.

Open Access This is an Open Access article distributed in accordance with the Creative Commons Attribution Non Commercial (CC BY-NC 4.0) license, which permits others to distribute, remix, adapt, build upon this work noncommercially, and license their derivative works on different terms, provided the original work is properly cited and the use is non-commercial. See: http:// creativecommons.org/licenses/by-nc/4.0/

\section{REFERENCES}

1. Doyle JJ. Chemotherapy and cardiotoxicity in older breast cancer patients: a population-based study. J Clin Oncol 2005;23:8597-605.

2. Armstrong GT, Oeffinger $\mathrm{KC}$, Chen $\mathrm{Y}$, et al. Modifiable risk factors and major cardiac events among adult survivors of childhood cancer. J Clin Oncol 2013;31:3673-80.

3. Mackey JR, Martin M, Pienkowski T, et al. Adjuvant docetaxel, doxorubicin, and cyclophosphamide in node-positive breast cancer: 10-year follow-up of the phase 3 randomised BCIRG 001 trial. Lancet Oncol 2013;14:72-80.

4. Wang L, Tan TC, Halpern EF, et al. Major cardiac events and the value of echocardiographic evaluation in patients receiving anthracycline-based chemotherapy. Am J Cardiol 2015;116:442-6.

5. Thavendiranathan P, Poulin F, Lim KD, et al. Use of myocardial strain imaging by echocardiography for the early detection of cardiotoxicity in patients during and after cancer chemotherapy. J Am Coll Cardiol 2014;63:2751-68.

6. Yu AF, Ky B. Roadmap for biomarkers of cancer therapy cardiotoxicity. Heart Br Card Soc 2016;102:425-30.

7. St John Sutton M, Pfeffer MA, Moye L, et al. Cardiovascular death and left ventricular remodeling two years after myocardial infarction: baseline predictors and impact of long-term use of captopril: information from the Survival and Ventricular Enlargement (SAVE) trial. Circulation 1997;96:3294-9.

8. Altena R, Perik PJ, Van Veldhuisen DJ, et al. Cardiovascular toxicity caused by cancer treatment: strategies for early detection. Lancet Oncol 2009;10:391-9.

9. Ky B, French B, May Khan A, et al. Ventricular-arterial coupling, remodeling, and prognosis in chronic heart failure. J Am Coll Cardio 2013;62:1165-72.

10. Kalam K, Otahal P, Marwick TH. Prognostic implications of global LV dysfunction: a systematic review and meta-analysis of global longitudinal strain and ejection fraction. Heart 2014;100:1673-80.

11. Zhang KW, French B, Khan AM, et al. Strain improves risk prediction beyond ejection fraction in chronic systolic heart failure. J Am Heart Assoc 2014;3:e000550.

12. Borlaug BA, Kass DA. Invasive hemodynamic assessment in heart failure. Heart Fail Clin 2009;5:217-28.
13. Narayan HK, French B, Khan A, et al. Noninvasive measures of ventricular-arterial coupling and circumferential strain predict cancer therapeutics-related cardiac dysfunction. JACC Cardiovasc Imaging 2016;9:1131-41.

14. Lang RM, Badano LP, Mor-Avi V, et al. Recommendations for cardiac chamber quantification by echocardiography in adults: an update from the American Society of Echocardiography and the European Association of Cardiovascular Imaging. J Am Soc Echocardiogr 2015;28:1-39.e14.

15. Farsalinos KE, Daraban AM, Ünlü S, et al. Head-to-head comparison of global longitudinal strain measurements among nine different vendors: the EACVI/ASE inter-vendor comparison study. $J$ Am Soc Echocardiogr 2015;28:1171-81,e2

16. Chen $\mathrm{CH}$, Fetics $\mathrm{B}$, Nevo $\mathrm{E}$, et al. Noninvasive single-beat determination of left ventricular end-systolic elastance in humans. J Am Coll Cardiol 2001;38:2028-34.

17. Piccart-Gebhart MJ, Procter M, Leyland-Jones B, et al. Trastuzumab after adjuvant chemotherapy in HER2-positive breast cancer. $N$ Eng $J$ Med 2005;353:1659-72.

18. Cardinale D, Colombo A, Bacchiani G, et al. Early detection of anthracycline cardiotoxicity and improvement with heart failure therapy. Circulation 2015;131:1981-8.

19. Chow EJ, Chen Y, Kremer LC, et al. Individual prediction of heart failure among childhood cancer survivors. J Clin Oncol 2015;33:394-402.

20. Cheng S, Larson MG, McCabe EL, et al. Age-and sex-based reference limits and clinical correlates of myocardial strain and synchrony: the Framingham Heart Study. Circ Cardiovasc Imaging 2013;6:692-9.

21. Borlaug BA, Redfield MM, Melenovsky V, et al. Longitudinal changes in left ventricular stiffness: a community-based study. Circ Heart Fail 2013;6:944-52.

22. Sawaya H, Sebag IA, Plana JC, et al. Early detection and prediction of cardiotoxicity in chemotherapy-treated patients. Am J Cardiol 2011;107:1375-80.

23. Sawaya H, Sebag IA, Plana JC, et al. Assessment of echocardiography and biomarkers for the extended prediction of cardiotoxicity in patients treated with anthracyclines, taxanes, and trastuzumab. Circ Cardiovasc Imaging 2012;5: 596-603.

24. Negishi K, Negishi T, Hare JL, et al. Independent and incremental value of deformation indices for prediction of trastuzumab-induced cardiotoxicity. J Am Soc Echocardiogr 2013;26:493-8.

25. Fallah-Rad N, Walker JR, Wassef $A$, et al. The utility of cardiac biomarkers, tissue velocity and strain imaging, and cardiac magnetic resonance imaging in predicting early left ventricular dysfunction in patients with human Epidermal growth factor receptor II-positive breast cancer treated with adjuvant trastuzumab therapy. J Am Coll Cardiol 2011;57:2263-70.

26. St John Sutton M, Cerkvenik J, Borlaug BA, et al. Effects of cardiac resynchronization therapy on cardiac remodeling and contractile function: results from Resynchronization Reverses Remodeling in Systolic Left Ventricular Dysfunction (REVERSE). J Am Heart Assoc 2015;4:e002054.

27. Hare JL, Brown JK, Leano R, et al. Use of myocardial deformation imaging to detect preclinical myocardia dysfunction before conventional measures in patients undergoing breast cancer treatment with trastuzumab. Am Heart $J$ 2009;158:294-301.

28. Wang J, Khoury DS, Yue Y, et al. Preserved left ventricular twist and circumferential deformation, but depressed longitudinal and radial deformation in patients with diastolic heart failure. Eur Heart $J$ 2007;29:1283-9.

29. Modesto K, Sengupta PP. Myocardial mechanics in cardiomyopathies. Prog Cardiovasc Dis 2014;57:111-24.

30. Hung C-L, Verma A, Uno $\mathrm{H}$, et al. Longitudinal and circumferential strain rate, left ventricular remodeling, and prognosis after myocardial infarction. J Am Coll Cardiol 2010;56: 1812-22.

31. Carasso S, Mutlak D, Lessick J, et al. Symptoms in severe aortic stenosis are associated with decreased compensatory circumferential myocardial mechanics. J Am Soc Echocardiogr 2015;28:218-25.

32. Hor KN, Wansapura J, Markham LW, et al. Circumferential strain analysis identifies strata of cardiomyopathy in Duchenne muscular dystrophy. J Am Coll Cardiol 2009;53:1204-10.

33. Nagata $\mathrm{Y}$, Takeuchi M, Mizukoshi K, et al. Intervendor variability of two-dimensional strain using vendor-specific and vendor-independent software. J Am Soc Echocardiogr 2015;28:630-41. 